\title{
Multilayer Membranes of Glycosaminoglycans and Collagen I Biomaterials Modulate the Function and Microvesicle Release of Endothelial Progenitor Cells
}

\author{
Bingyan Dai, ${ }^{1}$ Qunwen Pan, ${ }^{1}$ Zhanghua $\mathrm{Li}^{2},{ }^{2}$ Mingyan Zhao, \\ Xiaorong Liao, ${ }^{1}$ Keng $\mathrm{Wu}^{4}{ }^{4}$ and Xiaotang $\mathrm{Ma}^{1}$ \\ ${ }^{1}$ Guangdong Key Laboratory of Age-Related Cardiac and Cerebral Diseases, Affiliated Hospital of Guangdong Medical University, \\ Zhanjiang 524001, China \\ ${ }^{2}$ Department of Orthopaedics, Wuhan Third Hospital, Wuhan, Hubei 430000, China \\ ${ }^{3}$ Department of Stem Cell Research Center, Affiliated Hospital of Guangdong Medical University, Zhanjiang 524001, China \\ ${ }^{4}$ Department of Cardiology, Affiliated Hospital of Guangdong Medical University, Zhanjiang 524001, China
}

Correspondence should be addressed to Keng Wu; zjwukeng@hotmail.com and Xiaotang Ma; mxtgdmc@163.com

Received 4 February 2016; Revised 18 March 2016; Accepted 20 March 2016

Academic Editor: Yaoliang Tang

Copyright (C) 2016 Bingyan Dai et al. This is an open access article distributed under the Creative Commons Attribution License, which permits unrestricted use, distribution, and reproduction in any medium, provided the original work is properly cited.

\begin{abstract}
Multilayer composite membrane of biomaterials can increase the function of adipose stem cells or osteoprogenitor cells. Recent evidence indicates endothelial progenitor cells (EPCs) and EPCs released microvesicles (MVs) play important roles in angiogenesis and vascular repair. Here, we investigated the effects of biomaterial multilayer membranes of hyaluronic acid (HA) or chondroitin sulfate (CS) and Collagen I (Col I) on the functions and MVs release of EPCs. Layer-by-layer (LBL) technology was applied to construct the multilayer composite membranes. Four types of the membranes constructed by adsorbing either HA or CS and Col I alternatively with different top layers were studied. The results showed that all four types of multilayer composite membranes could promote EPCs proliferation and migration and inhibit cell senility, apoptosis, and the expression of activated caspase-3. Interestingly, these biomaterials increased the release and the miR-126 level of EPCs-MVs. Moreover, the CS-Col I membrane with CS on the top layer showed the most effects on promoting EPCs proliferation, EPCs-MV release, and miR-126 level in EPCs-MVs. In conclusion, HA/CS and Collagen I composed multilayer composite membranes can promote EPCs functions and release of miR-126 riched EPCs-MVs, which provides a novel strategy for tissue repair treatment.
\end{abstract}

\section{Introduction}

Medical biomaterials for the repair or replacement of tissue or organs are polymeric substances. Biomaterials like hyaluronic acid (HA), chondroitin sulfate (CS), and Collagen (Col) have biological activities on stem cells [1]. Fibril-forming Col I (Col I) as one of the most abundant matrix proteins can also affect the growth, spread, and differentiation of stem cells [2-4]. Glycosaminoglycans like HA and CS, as the extracellular matrix (ECM) components cooperating with numerous cell surface adhesive proteins (e.g., fibronectin) and cytokines (growth factors), can impinge on cell biological processes such as growth and differentiation $[5,6]$. Moreover, CS can promote the organization of soluble Col I precursors into fibrillar form which is essential for ECM structure and function, affecting the activity of adipose stem cells [7].

Various surface modification techniques have been used to improve the bioactivity of biomaterials. The so-called layer-by-layer (LBL) technique has emerged as a simple and versatile method for physical surface modification. Macromolecules including Col I, HA, and CS have natural adsorption and adhesion characters which allow paving multilayer composite membrane. Col I and either CS or HA are considered to be suitable for making multilayer membranes that have ECM-like characteristics and functions $[8,9]$. The LBL multilayer film with HA-Col I or CS-Col I as raw materials could modulate fibroblast cell and osteoprogenitor stem cell functions (proliferation, migration, and regeneration) and 
might be used for making the bioactive coatings of implants $[1,10]$. However, whether the components (HA-Col I or CS-Col I) and top layer (HA, CS, or Col I) of multilayer biomaterial membranes affect EPC activities remains unclear.

Endothelial progenitor cells (EPCs) are precursors of endothelial cells mobilized from bone marrow to peripheral blood in response to ischemia or injury $[11,12]$. In recent years, many groups have reported the therapeutic effects of EPCs on tissue wound, heart infarction, and ischemic stroke [12-15]. It has been suggested that the therapeutic effects of EPCs are likely affected by pathological conditions of the body $[16,17]$. Therefore, enhancing EPC function is a reasonable strategy to be applied for the treatment. Microvesicles (MVs) are lipid membrane vesicles with diameter of 0.1$1 \mu \mathrm{m}$ which are released when cells are activated or under apoptosis. They carry the characteristic of their mother cells and affect the function of recipient cells by transporting the contents of proteins, miRNAs (miRs), and mRNAs [18]. The therapeutic effects of EPCs are currently considered to be partly related to EPCs-MVs [19]. Moreover, it has been shown that miR-126 plays an important role in mediating EPCs-MV functions [20].

In this study, we investigated the effects of different multilayer membranes (HA-Col I or CS-Col I) with different top layers (HA, CS, or Col I) on EPC cell proliferation, migration, apoptosis, senescence, and MV release and miR126 carry.

\section{Materials and Methods}

2.1. Preparation of Multilayer Composite Membranes. Multilayer membranes were fabricated on cleaned culture dish, respectively. Polyethyleneimine (PEI) was used at $\mathrm{pH} 7.4$ as anchoring base layer to obtain a positive net charge of the respective substrates. Multilayers were formed on top of the PEI layer using CS or HA as polyanion and Col I as polycation. The polyanion layer (HA or CS) was adsorbed for $15 \mathrm{~min}$ at $\mathrm{pH}$ 4.0, while Col I layer was adsorbed for $20 \mathrm{~min}$ at the same $\mathrm{pH}$ value. Each adsorption step was followed by rinsing with $0.15 \mathrm{M}$ sodium chloride solution $(\mathrm{pH} 4.0$ ) for $3 \times 5 \mathrm{~min}$. As a result, biomaterial membrane systems with multilayers were obtained by adsorbing HA/CS and Col I alternatively on top of the PEI layer. We constructed four types of multilayer membranes. They were type 1 (defined as $\mathrm{HA}^{*}$-Col I): three bilayers of HA-Col I with an additional layer of HA (seven alternative layers of HA and Col I with HA on the top), type 2 (defined as HA-Col I*): four bilayers of HA-Col I (eight alternative layers of HA and Col I with Col I on the top), type 3 (defined as $\mathrm{CS}^{*}$-Col I): three bilayers of CS-Col I with an additional seventh layer of CS (seven alternative layers of CS and Col I with CS on the top), and type 4 (defined as CS-Col I*): four bilayers of CS-Col I (eight alternative layers of CS and Col I with Col I on the top). Untreated well plate was set as the vehicle group.

2.2. Culture of EPCs. Male adult (8-10 weeks of age; weight ranges from 20 to $25 \mathrm{~g}$ ) $\mathrm{C} 57 / \mathrm{Bl}$ mice were used for all experiments. EPCs were generated from bone marrow mononuclear cells (MNCs) as we previously reported [21, 22]. In brief, bone marrow was flushed out from tibias and femurs and MNCs were isolated by using density gradient centrifuge method. MNCs isolated from C57 mice were counted and plated on fibronectin-coated 6-well plates and then grown in endothelial cell basal medium-2 (EBM-2) supplemented with 5\% FCS containing EPC growth cytokine cocktail (Lonza, Walkersville, MD, USA). After 3-day culture in wells not coated (vehicle group) or coated with biomaterial membranes, nonadherent cells were removed by washing with PBS. Thereafter, culture medium was changed every 2 days.

2.3. Cell Apoptosis Assay. Cell apoptosis was analyzed by Hoechst 33258 staining and Annexin V-PE/7-AAD apoptosis detection kit (BD Biosciences) as we previously described [23]. In brief, EPCs were seeded on culture wells not coated (vehicle group) or coated with biomaterial $\mathrm{HA}^{*}$-Col I, HACol I* CS $^{*}$-Col I, or CS-Col I* membrane at a density of $2 \times$ $10^{5} /$ well in $2 \mathrm{~mL}$ serum-free EBM-2 medium. After culture for $24 \mathrm{~h}$, cell apoptosis rates were measured. For Hoechst 33258 staining, cells were fixed and stained with Hoechst 33258 solution according to the manufacturer's instructions (Beyotime) followed by fluorescence microscope observation. Five independent fields were assessed for each well. The average number of positive cells and total cells per field (magnification, 200x) were determined. The apoptotic rate of cells was defined as the ratio of positive cells versus total cells. And for Annexin V-PE/7-AAD apoptosis detection, cells were washed with PBS, resuspended with $100 \mu \mathrm{L} 1 \mathrm{x}$ Annexinbinding buffer, incubated with $5 \mu \mathrm{L}$ PE-conjugated Annexin $\mathrm{V}$ and $5 \mu \mathrm{L}$ 7-aminoactinomycin (7-AAD) for $15 \mathrm{~min}$ in the dark, and then analyzed by flow cytometry. Cells stained with both Annexin V-PE and 7-AAD were considered to be late apoptotic EPCs, and those stained only with Annexin V-PE were considered to be early apoptotic cells. The experiment was repeated three times. And three plates per experiment were analyzed in each group.

2.4. EPCs Proliferation Assay. Proliferative capability of EPCs was tested by MTT (3-[4,5-dimethylthiazol-2-yl]-2,5diphenyltetrazolium bromide) (Sigma, $5 \mathrm{mg} / \mathrm{mL}$ ) assay. EPCs were seeded in 96-well plate not coated or coated with biomaterial membranes (HA* ${ }^{*} \mathrm{Col} \mathrm{I,} \mathrm{HA-Col} \mathrm{I*}, \mathrm{CS}^{*}$-Col I, or CS-Col I*) and cultured in $100 \mu \mathrm{L}$ EBM-2 (supplemented with $10 \%$ FBS $)$ as described above. MTT solution $(20 \mu \mathrm{L})$ was added and incubated with cells for $4 \mathrm{~h}$ at $37^{\circ} \mathrm{C}$; then $150 \mu \mathrm{L}$ DMSO was added to each well and incubated with the cells for $15 \mathrm{~min}$ at $37^{\circ} \mathrm{C}$. The optical density (OD) value of cells was read at $490 \mathrm{~nm}$ in a microplate reader (BioTek). Cells in triplicate wells were examined at each time point, and the experiment was repeated for three times. Results were calculated from the values obtained in three independent experiments.

2.5. Migration Assay of EPCs. The migration distance of EPCs was measured by scratch assay. EPCs were grown to confluence on 6-well cell culture plate not coated (vehicle group) or coated with biomaterial membranes ( $\mathrm{HA}^{*}$-Col I, 
HA-Col I*, CS* ${ }^{*}$-Col I, or CS-Col I* ${ }^{*}$. A scratch was made through the cell monolayer using a P200 pipette tip. After washing with PBS, cells were cultured in $0.5 \%$ FBS maintenance medium. Photographs of the wounded area were taken immediately $(0 \mathrm{~h})$ and $48 \mathrm{~h}$ after making the scratch to monitor the invasion of cells into the scratched area. Quantitative analysis of migration was measured as the following equation:

(cell free area at $0 \mathrm{~h}-$ cell free area at $16 \mathrm{~h}$ ) cell free area at $0 \mathrm{~h}$

2.6. Cell Senescence Assay. Long-term culture was used to establish the senescent model of EPCs. In brief, EPCs were cultured in 6-well plate not coated (vehicle group) or coated with biomaterial membranes (HA ${ }^{*}$-Col I, HA-Col I*, $\mathrm{CS}^{*}$ Col I, or CS-Col I*) for 4 days. After that, $\beta$-Galactosidase Staining Kit (Beyotime, China) was used to analyze the senescent EPCs. In brief, after washing with PBS, EPCs grown on 6-well plate not coated (vehicle group) or coated with biomaterial membranes $\left(\mathrm{HA}^{*}\right.$-Col I, HA-Col I* ${ }^{*} \mathrm{CS}^{*}$-Col I, or CS-Col I*) were treated with $2 \%$ formaldehyde and $0.2 \%$ glutaraldehyde in PBS for $6 \mathrm{~min}$ and then incubated with fresh X-gal staining solution $(1 \mathrm{mg} / \mathrm{mL} \mathrm{X-gal,} 5 \mathrm{mmol} / \mathrm{L}$ potassium ferrocyanide, $5 \mathrm{mmol} / \mathrm{L}$ potassium ferricyanide, and $2 \mathrm{mmol} / \mathrm{L} \mathrm{MgCl}_{2} ; \mathrm{pH}$ 6) for $12 \mathrm{~h}$ at $37^{\circ} \mathrm{C}$ without $\mathrm{CO}_{2}$. After staining, blue-stained cells and total cells were counted at 3 different microscopic fields. The percentage of $\beta$-galactosidase positive cells was calculated.

2.7. Preparation and Detection of EPCs-MVs. EPCs-MVs were generated from EPCs cultured as previously described [20]. In brief, EPCs were cultured in cell culture dishes not coated (vehicle group) or coated with biomaterial membranes (HA ${ }^{*}$-Col I, HA-Col I* ${ }^{*} \mathrm{CS}^{*}$-Col I, or CS-Col I*). When grown to $80 \%$ confluence, cells were washed with PBS and cultured in fresh medium for $48 \mathrm{~h}$. Then the cell medium was collected and centrifuged at $300 \mathrm{~g}, 15 \mathrm{~min}$, followed by $2000 \mathrm{~g}$, $30 \mathrm{~min}$, to remove cell debris. The cell-free culture medium was centrifuged at $20,000 \mathrm{~g}, 2 \mathrm{~h}$ to pellet MVs. The MVs were resuspended with $20 \mathrm{~nm}$ filtered (Whatman, Pittsburgh, PA) PBS. The number of cell particles was detected by the nanoparticle analyzer (NTA300, Malvern, Britain).

2.8. Analysis of miR-126. The level of miR-126 in EPCMVs was determined by real-time PCR. Total miRs were extracted by using miRNeasy Mini kit (QIAGEN) following manufacturer's instructions. The miR-126 cDNAs were synthesized using Hairpin-it ${ }^{\mathrm{TM}}$ miRNAs RT-PCR Quantization kit (GenePharma, Shanghai, China) based on manufactory instruments $\left(25^{\circ} \mathrm{C}\right.$ for $30 \mathrm{~min}, 42^{\circ} \mathrm{C}$ for $30 \mathrm{~min}$, and $85^{\circ} \mathrm{C}$ for $5 \mathrm{~min}$ ). Real-time PCR parameters were $95^{\circ} \mathrm{C}$ for $3 \mathrm{~min} ; 40$ cycles were performed at $95^{\circ} \mathrm{C}$ for $12 \mathrm{~s}$ and $60^{\circ} \mathrm{C}$ for $40 \mathrm{~s}$ [23]. PCR primers are as follows: 5-TATGGTTGTTCTCGACTCCTTCAC-3 and 5-TCGTCTGTCGTACCGTGAGTAAT3 for miR-126; 5-CTC GCT TCG GCA GCA CA-3 and 5AAC GCT TCA CGA AYY YGC GT-3 for U6. Quantitative real-time PCR was conducted on a real-time PCR system
(Bio-Rad). Small nuclear RNA U6 (U6) was used as an internal control. Relative expression of miR-126 was calculated by using the $2^{-\Delta \Delta \mathrm{CT}}$ method [24].

2.9. Western Blot Analysis. Proteins from EPCs were extracted with lysis buffer. Protein lysates were electrophoresed through SDS-PAGE gel and transferred onto PVDF membranes. The membranes were blocked for $1 \mathrm{~h}$ and incubated with primary antibodies against caspase-3 (CST, USA) and $\beta$-actin (CST, USA) at $4^{\circ} \mathrm{C}$ overnight. After washing 3 times for 30 min with TBST, the immunoreactivity was visualized by ECL solution (GE Healthcare, USA).

2.10. Statistical Analysis. Data were all expressed as the mean \pm SD. Multiple comparisons were performed by two-way ANOVA. Comparisons for two groups were performed by using Student's $t$-test (GraphPad Prism 5 software). $p<0.05$ was considered significant.

\section{Results}

3.1. Multilayer HA/CS-Col I Membranes Similarly Inhibit EPC Apoptosis. Hoechst 33258 staining and Annexin V$\mathrm{PE} / 7-\mathrm{AAD}$ analysis revealed that multilayer composite membranes significantly decreased serum-free culture induced EPC apoptosis (versus vehicle; Figures 1(a) and 1(b)). In addition, western blot data showed that the cleaved caspase3 level, which was associated with induction of apoptosis, was inhibited by multilayer composite membranes (versus vehicle; Figure 1(c)). Interestingly, we did not find any significant differences of those measurements among the four multilayer composite membranes, indicating that all of them have antiapoptotic effects on EPCs.

3.2. Multilayer HA/CS-Col I Membranes Promote EPC Proliferation, with the Best Seen in CS*-Col I Membrane. MTT assay showed that all four multilayer composite membranes $\left(\mathrm{HA}^{*}\right.$-Col I, HA-Col I* ${ }^{*} \mathrm{CS}^{*}$-Col, and CS-Col I*) markedly promoted EPC proliferation (1.6 $\pm 0.06-, 1.9 \pm 0.13-, 2.3 \pm 0.22-$, and $1.7 \pm 0.96$-fold vehicle, resp.; Figure 2) when compared with untreated group. Meanwhile, we found that the $\mathrm{CS}^{*}$ Col was most effective on increasing EPC proliferation. The OD490 value of CS ${ }^{*}$-Col I treated EPCs was $1.39 \pm 0.11$-, $1.17 \pm 0.07$-, and $1.32 \pm 0.08$-fold HA ${ }^{*}$-Col I, HA-Col I* , and CS-Col I* treated EPCs, respectively (Figure 2).

\subsection{Multilayer HA/CS-Col I Membranes Similarly Increase} EPC Migration. A scrape injury assay was carried out to assess the effect of multilayer composite membranes on EPC migration. The result revealed that all four multilayer composite membranes significantly increased the migration ability of EPCs, with the average migration area increased by $1.88 \pm 0.15-, 1.72 \pm 0.051-, 1.91 \pm 0.23-$, and $1.61 \pm 0.062-$ fold vehicle, respectively, for $\mathrm{HA}^{*}$-Col I, HA-Col I* ${ }^{*} \mathrm{CS}^{*}$ Col, and CS-Col I* treated EPCs (Figure 3). However, we did not find any difference among the four multilayer composite membranes. 

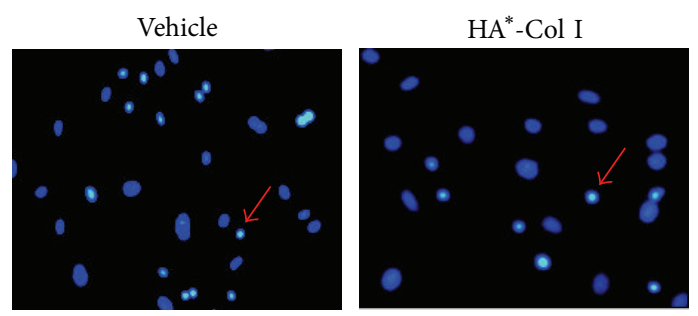

HA-Col I*
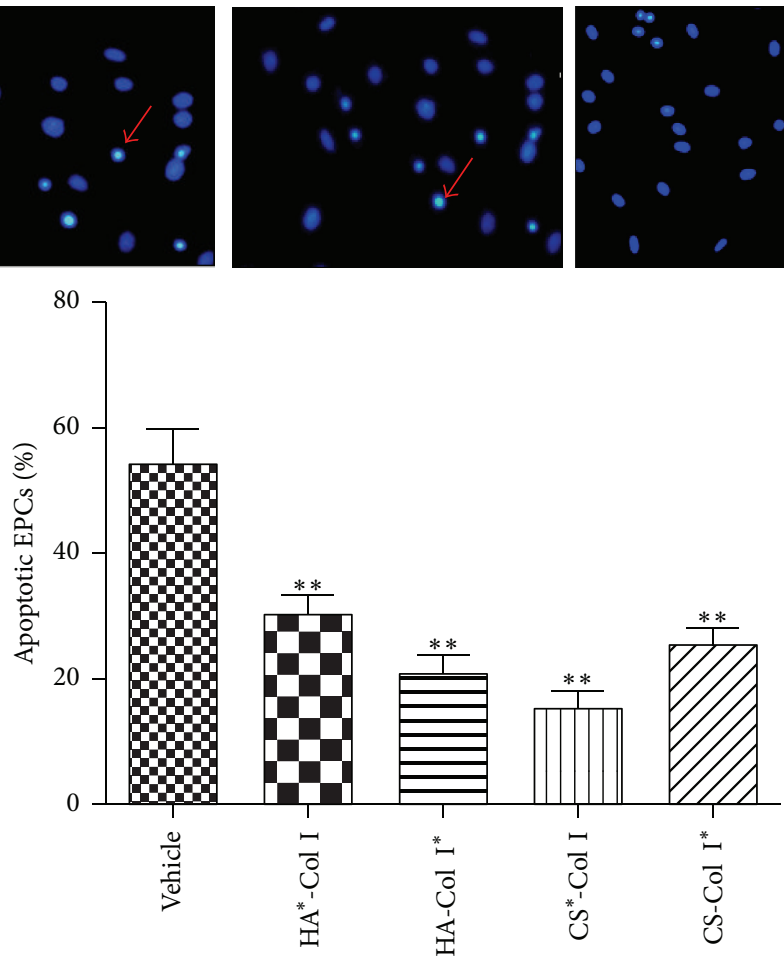

(a)
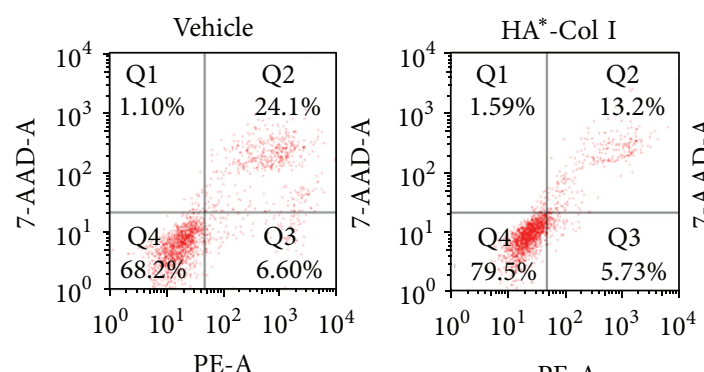

PE-A
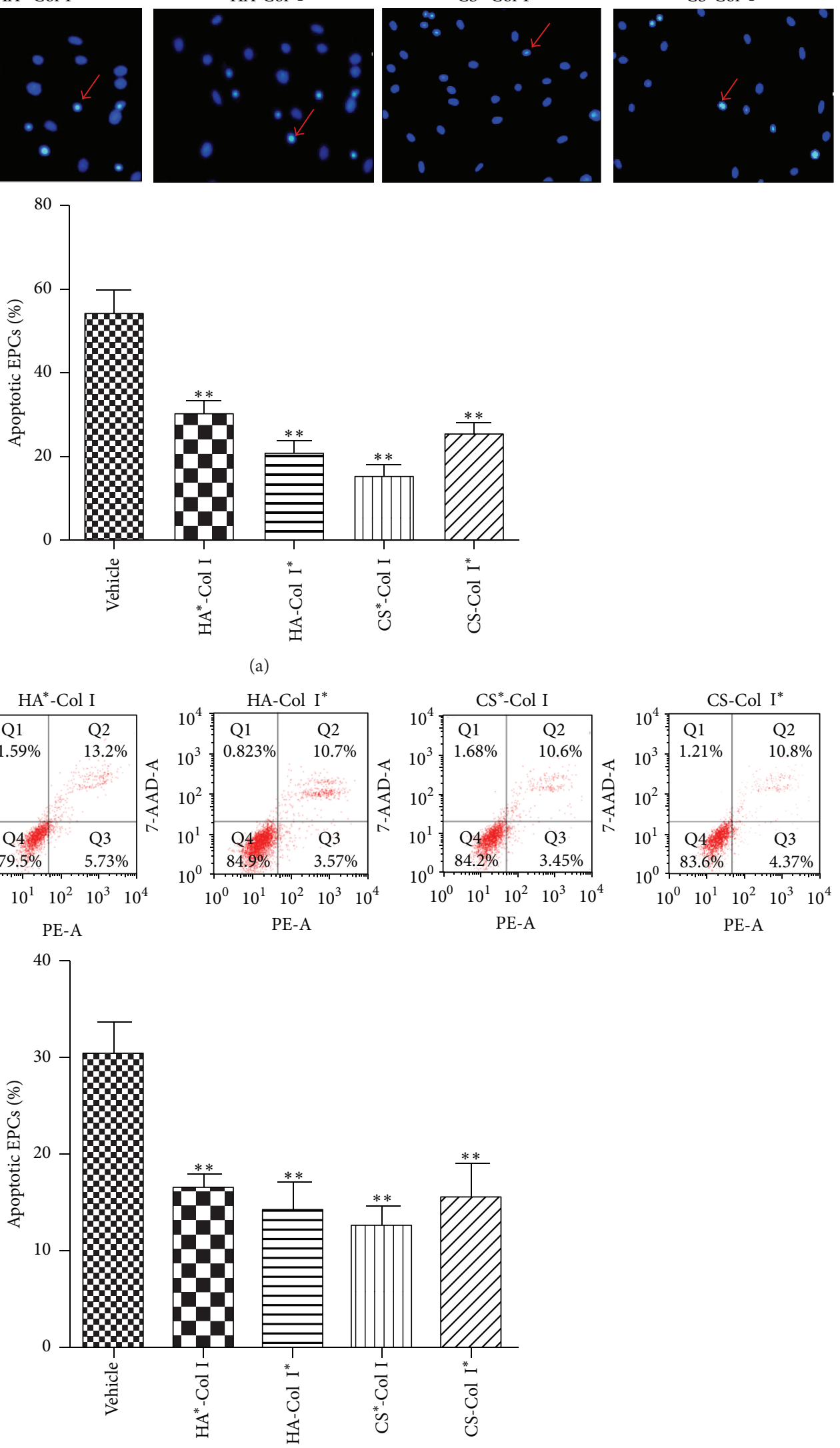

(b)

FIgure 1: Continued. 


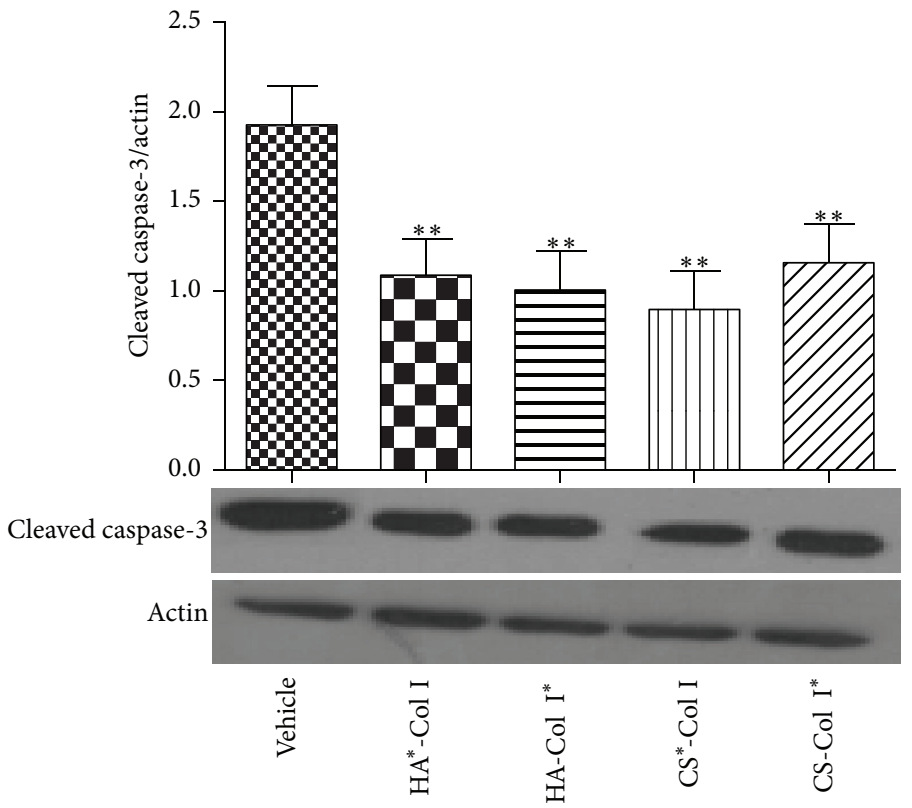

(c)

FIGURE 1: Effects of multilayer composite biomaterial membranes on EPC apoptosis. (a) EPC apoptosis determined by Hoechst 33258 staining (red arrows). (b) EPC apoptosis determined by Annexin V-PE/7-AAD staining and flow cytometric analysis. (c) Cleaved caspase-3 expression detected by western blot. ${ }^{* *} p<0.01$ versus vehicle, $n=3$ /group.

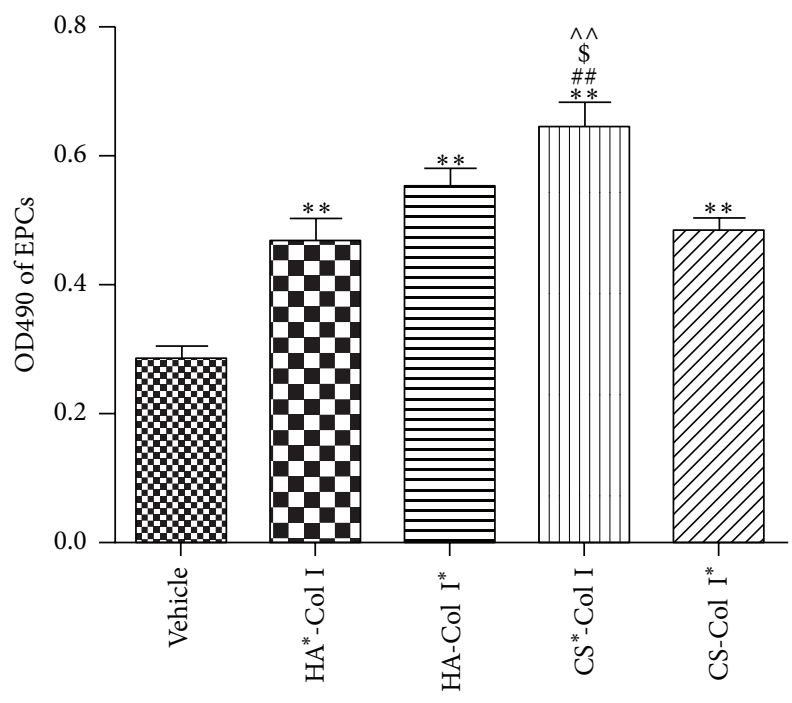

FIGURE 2: Effects of multilayer composite biomaterial membranes on the proliferation of EPCs. CS*-Col I group showed the most significant effect on the EPC proliferation. ${ }^{* *} p<0.01$ versus vehicle, $\# p<0.01,{ }^{\$} p<0.05$, and ${ }^{M} p<0.01$ versus $\mathrm{HA}^{*}$-Col I, HA-Col I*, or CS-Col I*,$n=3$ /group.

3.4. Multilayer HA/CS-Col I Membranes Similarly Inhibit EPC Senescence. To determine the cellular aging, $\beta$-galactosidase was used as a biochemical marker. In our experiments, EPCs showed $32.33 \pm 3.05 \%$ senescence rate after long-term culture. As we expected, EPCs had reduced senescence rate when they grew on $\mathrm{HA}^{*}$-Col I, HA-Col I* ${ }^{*} \mathrm{CS}^{*}$-Col, and CS-Col $I^{*}$ multilayer composite membranes $(17.7 \pm 3 \%, 15.5 \pm 3 \%$, $14.2 \pm 1.51 \%$, and $15.8 \pm 3 \%$, resp., Figure 4$)$. In addition, we did not find any significant differences among the four groups.

3.5. Multilayer HA/CS-Col I Membranes Differently Increase EPC-MVs Releasing. Our results showed that EPCs growing on $\mathrm{HA}^{*}$-Col I, HA-Col I* ${ }^{*} \mathrm{CS}^{*}$-Col I, or CS-Col I* coated culture plate released more MVs (versus vehicle; Figure 5). We also found that the four types of membranes showed different effects on EPC-MV release. CS*-Col I had the highest effect on stimulating EPC-MV release (versus HA* Col I, HA-Col I*, or CS-Col I*; Figure 5). The MVs release activity of EPCs was also different in other groups (HA-Col $\mathrm{I}^{*}, \mathrm{HA}^{*}$-Col I, and CS-Col I* ; Figure 5), suggesting that EPCEM release is influenced by different biomaterials.

3.6. Multilayer HA/CS-Col I Membranes Differently Increase miR-126 Level in EPC-MVs. MV carried miRs could mediate the function of MVs. We found that the miR-126 in EPCMVs was increased by the four different membranes (HA* Col I, HA-Col I*, CS*-Col I, or CS-Col I*) after $24 \mathrm{~h}$ culture (Figure 6). We also found that effect of $\mathrm{CS}^{*}-\mathrm{Col}$ was the highest (versus $\mathrm{HA}^{*}$-Col I, HA-Col I* ${ }^{*}$ or CS-Col I*; Figure 6). The miR-126 level in EPC-MVs from HA-Col I* was much higher than that from $\mathrm{HA}^{*}$-Col I (versus $\mathrm{HA}^{*}$-Col I; Figure 6), and that from CS-Col I* was lower than that from $\mathrm{CS}^{*}$-Col I (versus CS-Col I* ; Figure 6).

\section{Discussion}

In this study, we studied the effects of four types of multilayer membranes (HA* ${ }^{*}$ Col I, HA-Col I* ${ }^{*} \mathrm{CS}^{*}-\mathrm{Col} \mathrm{I}$, and CS$\mathrm{Col} \mathrm{I}^{*}$ ) on EPC functions and EPC-MV release. The major 

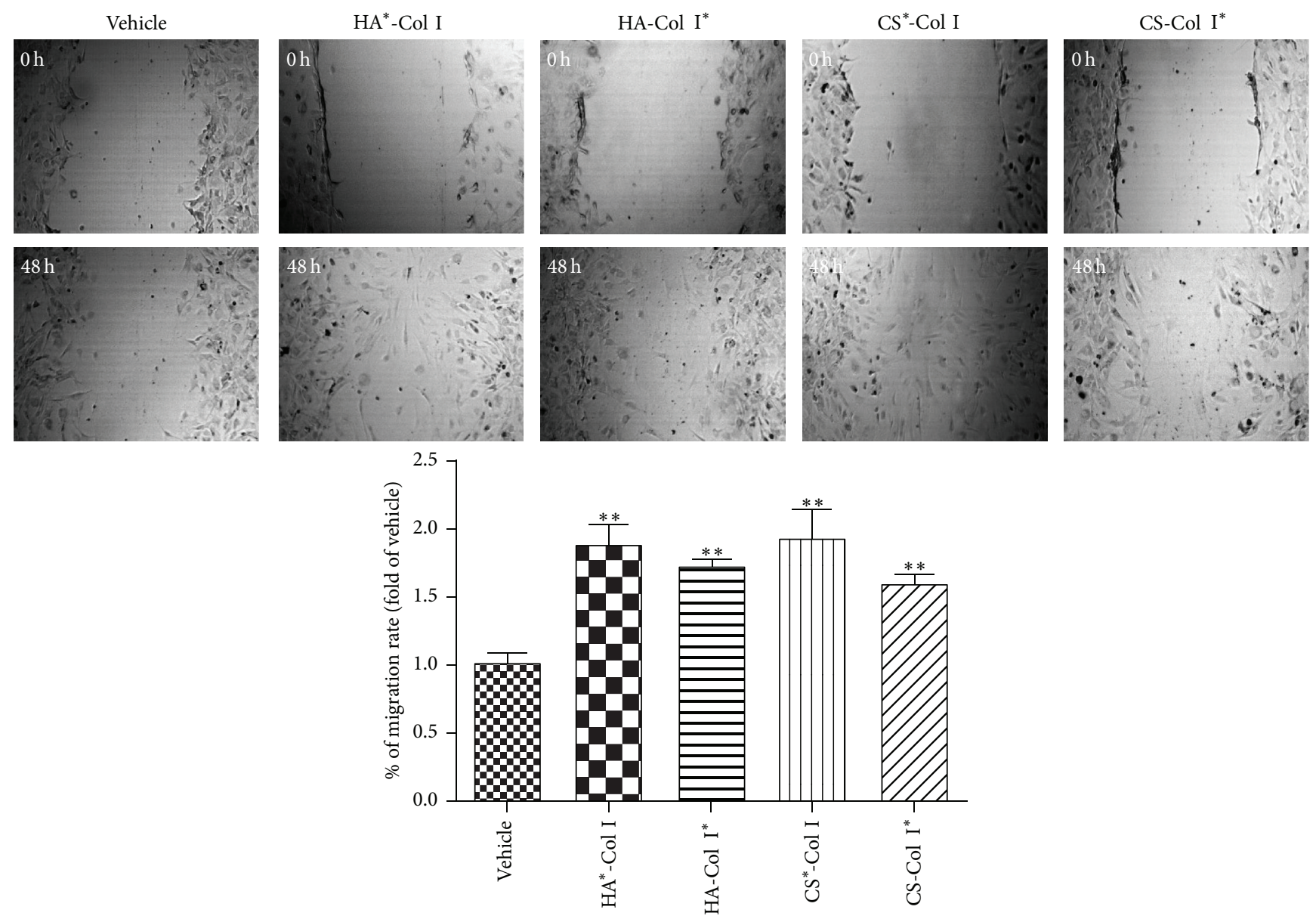

FIGURE 3: Effects of multilayer composite biomaterial membranes on the migration of EPCs. ${ }^{* *} p<0.01$ versus vehicle, $n=3 /$ group.

findings suggest that all the four types of membranes could promote EPC proliferation and migration and inhibit cell apoptosis and senility. The membranes could also promote the release of miR-126 rich EPC-MVs. Among them, the CS* Col I had the best effects on promoting EPC proliferation and miR-126 riched EPC-MV release.

Recent evidence suggests that surface modification with biomolecules like oligopeptides or glycans which can represent components of the ECM has been frequently applied to achieve an improved healing response of medical implants [25]. Biomaterial surfaces like glycosaminoglycans, such as HA and CS, have been found to enhance signal transduction into cells and promote osteoprogenitor cell spreading, proliferation, and differentiation $[2,3]$. In this study, we found that biomaterial multilayer membranes composed of (HA/CS) and Col I promoted EPCs function. Moreover, the proliferation promoting effects were significantly enhanced when CS was applied on the top of $\mathrm{CS}^{*}$-COI composed membrane, suggesting that CS might be more appropriate for EPCs.

EPCs are believed to play an important role in endothelial integrity, vascular homeostasis, and angiogenesis, which represents an important endogenous tissue repair and regenerative mechanism. Introduction or mobilization of EPCs can restore tissue vascularization after ischemic stroke and reestablish endothelial integrity $[21,26]$. Various studies have attempted to enhance EPC functions under physiological and pathological conditions, so that they can be effectively used for therapy $[27,28]$. Our group has reported that overexpression of CXCR4 and ACE2 could enhance the beneficial effect of EPCs-based therapy for ischemic stroke by promoting EPC proliferation and survival $[29,30]$. The present study for the first time provides novel evidence regarding the effects of biomaterial membranes (HA/CS and Col I multilayers) on EPCs, which might be important when biomaterials and stem cells are applied in clinical therapy. The beneficial effects of biomaterial membranes for EPCs are likely through promoting cell proliferative activity, inhibiting apoptosis, and decreasing cell senescence. Several pathways may be involved in these effects. For example, Ras/ERK/VEGF and PI3K/Akt/eNOS signal pathways have been reported to be related with EPC proliferation, migration, and tube formation abilities [27, 28]. However, the precise underlying mechanisms need to be further investigated.

The therapeutic effect of stem cells is partly associated with their released MVs [19]. MVs not only carry the characteristics of mother cell source and are used as biomarkers, but also affect the functions of recipient cells 

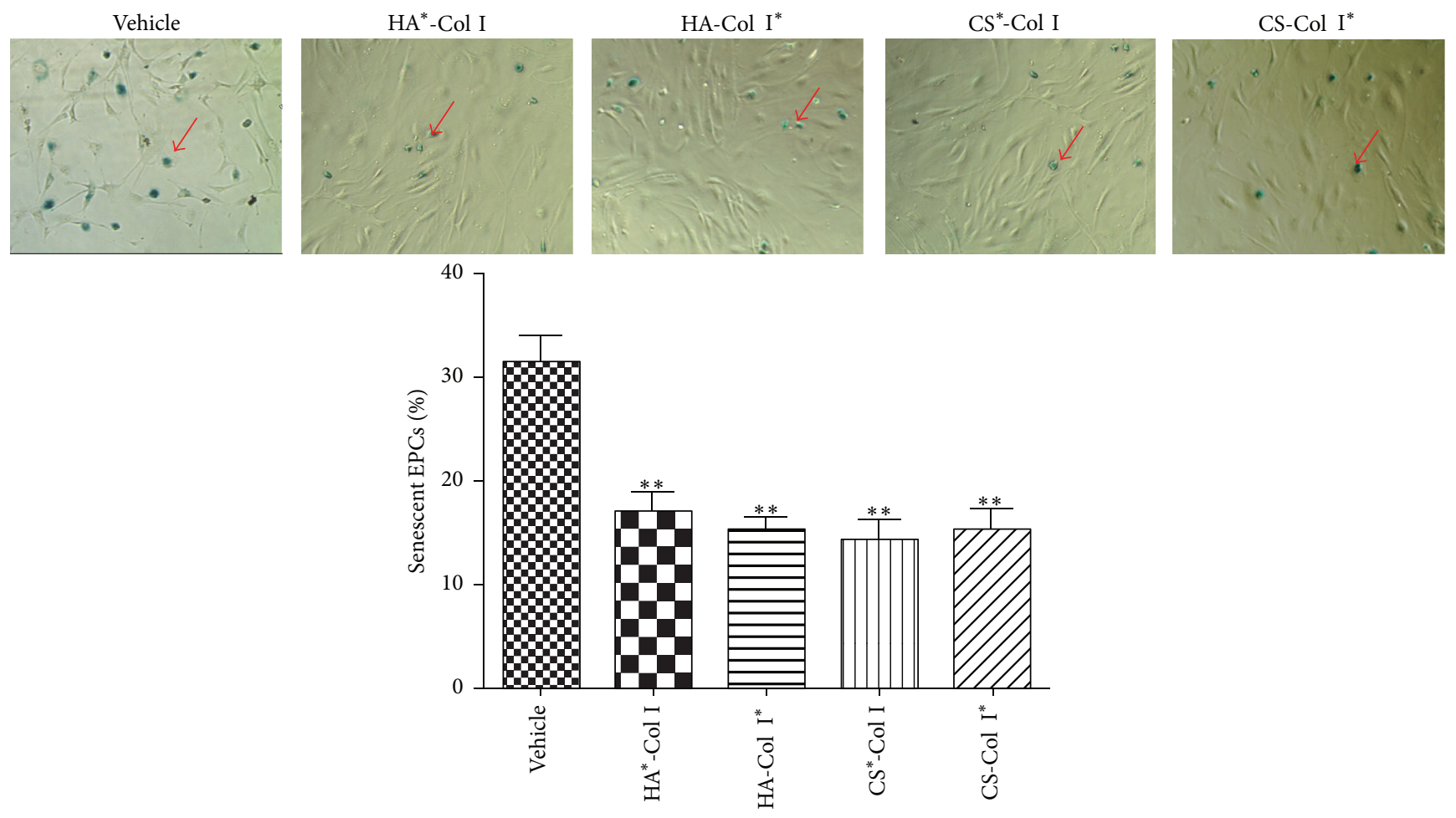

FIGURE 4: Effects of multilayer composite biomaterial membranes on senescent EPCs. Senescent EPC monitored by $\beta$-galactosidase staining (red arrows indicating apoptotic cells). ${ }^{* *} p<0.01$ versus vehicle, $n=3$ /group.

through transferring the contents (proteins, miRNAs, and mRNAs) [31]. The numbers of circulating endothelial cells released MVs were demonstrated to increase in many vascular diseases including severe hypertension, acute coronary syndromes, and various forms of vasculitis [18, 32-34]. We have reported that EPC-MVs were negatively correlated with stroke severity and infarct volume in brain ischemic patient. Moreover, the increased level of EPC-MVs positively associated with vascular density in ischemic stroke [20]. MVs could be important underlying the mechanisms by which EPCs exert their functions. EPC-MVs have been reported to protect the kidney from ischemia-reperfusion injury and could enhance neoangiogenesis of human pancreatic islets $[35,36]$. EPC-MVs could promote endothelial cell survival, proliferation, and tube formation ability, via the miR-126 in MVs [19], and play critical roles in angiogenesis and vascular integrity [37]. Circulating miR-126 which could exert an atheroprotective effect has been shown to be reduced in vascular diseases including stroke and coronary artery disease [38-40]. The miR-126 in EPC-MVs can be transferred to neighboring cells to modulate the activities of recipient cells. It was shown that miR-126 riched EPC-MVs ameliorated H/R induced HB-ECs apoptosis and dysfunction by activating PI3K/eNOS/NO pathway [20]. Jansen et al. reported that miR-126 was transported into human coronary artery endothelial cells (HCAECs) by endothelial cells released MVs (EMVs) and regulated HCAEC migration and proliferation [41]. These indicate that the level and carried contents of MVs are important factors closely related to the cell status and functions. EPC-MVs rich in miR-126 may exert beneficial effects on recipient cells. In the present study, we showed that the number and the miR-126 level of EPC-MVs from experimental multilayer membranes especially in $\mathrm{CS}^{*}$ COI were all much more than those of the control group, indicating that the studied biomaterial membranes could promote EPC-MVs functions. In other words, they may help enhance EPCs therapeutic effects. The multilayer treatments improved EPC functions may be not only through regulating proliferation, migration, senescence, and apoptosis of EPCs, but also by releasing miR-126 riched MVs further promoting neighboring cell functions. However, this presumption needs to be verified by further investigations.

In addition, the number and the miR-126 level of EPCMVs from membrane $\mathrm{HA}^{*}$-Col I were strongly different from those of membrane HA-Col I*. The number and the miR126 level of EPC-MVs from CS* -Col I were also significantly different from those of membrane CS-Col I*. Therefore, we speculate that biomaterial which directly contacts with the EPCs plays a key role in regulating the release and miR126 contents of EPC-MVs. This could help to choose the most effective biomaterial membranes for improving EPC functions when they are applied in clinical therapies.

\section{Conclusion}

In conclusion, biomaterials including $\mathrm{HA}^{*}$-Col I, HA-Col I*, $\mathrm{CS}^{*}$-Col I, or CS-Col I* constructed multilayer membranes can modulate EPC functions and the number and miR-126 level of EPC-MVs. The beneficial effects of these biomaterial membranes are likely through promoting the proliferative, 


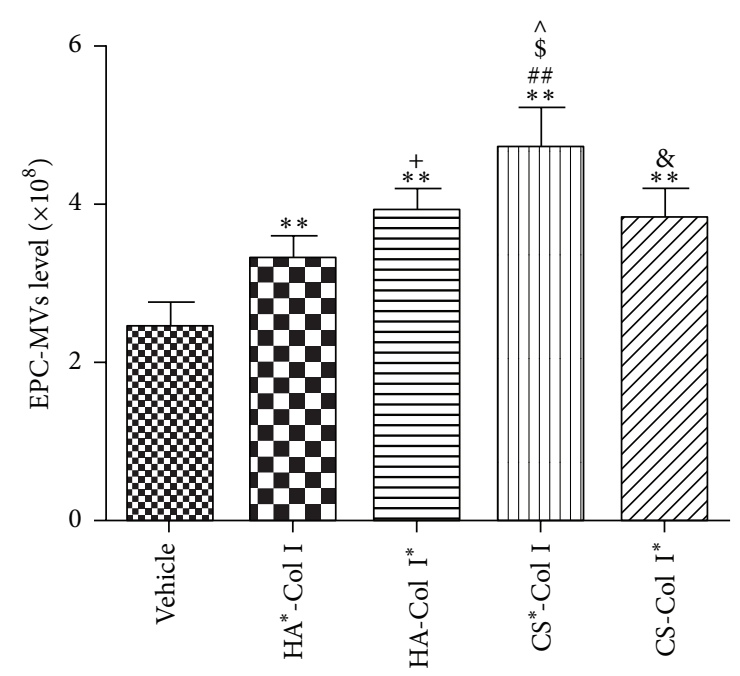

FIGURE 5: Effects of multilayer composite biomaterial membranes on the release of EPC-MVs. EPC-MVs level from CS* ${ }^{*}$-Col I group was higher than that from other biomaterial membrane groups. The level of EPC-MVs from HA-Col I* group was higher than that from $\mathrm{HA}^{*}$-Col I group, and EPC-MVs level from CS-Col I* group was lower than that from $\mathrm{CS}^{*}$-Col I group. ${ }^{* *} p<0.01$ versus vehicle, ${ }^{\# \#} p<0.01,{ }^{\$, \wedge} p<0.05$ versus $\mathrm{HA}^{*}$-Col I, HA-Col I* , or CS-Col $\mathrm{I}^{*},{ }^{+} p<0.05$ versus $\mathrm{HA}^{*}-\mathrm{Col} \mathrm{I}$, and ${ }^{\&} p<0.05$ versus $\mathrm{CS}^{*}-\mathrm{Col} \mathrm{I}$, $n=3 /$ group.

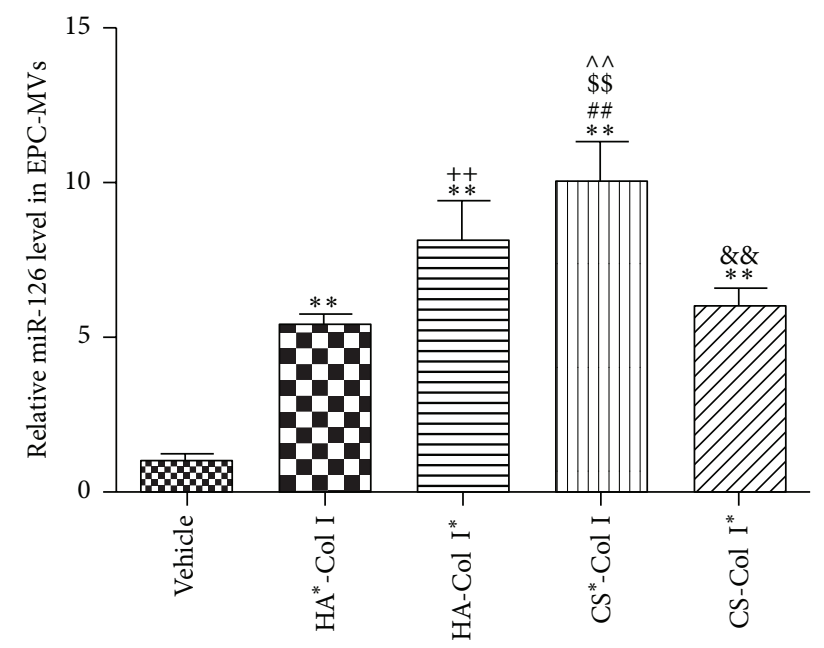

FIGURE 6: Effects of multilayer composite biomaterial membranes on the level of miR-126 in EPCs-MVs. miR-126 level in EPC-MVs from $\mathrm{CS}^{*}$-Col I group was the highest. The miR-126 level in EPCsMVs from HA-Col I* group was higher than that from $\mathrm{HA}^{*}$-Col I group, and miR-126 in EPCs-MVs from CS-Col I* group was lower than that from $\mathrm{CS}^{*}$-Col I group. ${ }^{* *} p<0.01$ versus vehicle, ${ }^{\#, \$ \$, \wedge \wedge} p<$ 0.01 versus $\mathrm{HA}^{*}$-Col I, HA-Col I* ${ }^{*}$, or CS-Col I*,${ }^{++} p<0.05$ versus $\mathrm{HA}^{*}-\mathrm{Col} \mathrm{I}$, and ${ }^{\& \&} p<0.05$ versus $\mathrm{CS}^{*}-\mathrm{Col} \mathrm{I}, n=3$ /group.

antiapoptotic, and antisenescence abilities of EPCs, as well as through enhancing EPC-MV release and miR-126 level. Therefore, combined use of biomaterials and stem cells may present a novel approach for promoting wound healing and tissue regeneration. However, further investigations in animal models and patients are required.

\section{Competing Interests}

The authors declare that they have no competing interests.

\section{Authors' Contributions}

Bingyan Dai, Qunwen Pan, and Zhanghua Li contributed equally to this work.

\section{Acknowledgments}

This work was supported by National Natural Science Foundation of China (NSFC, nos. 81400360 and 81472103); Science and Technology Planning Project of Guangdong Province (no. 2014A020212293); Medical Scientific Research Foundation of Guangdong Province (no. B2014311); and National Natural Science Foundation of Hubei Province (no. 2015CFA079).

\section{References}

[1] M. Zhao, L. Li, C. Zhou et al., "Improved stability and cell response by intrinsic cross-linking of multilayers from collagen i and oxidized glycosaminoglycans," Biomacromolecules, vol. 15, no. 11, pp. 4272-4280, 2014.

[2] P. Rico, J. C. Rodriguez Hernandez, D. Moratal, G. Altankov, M. Monleon Pradas, and M. Salmerón-Sánchez, "Substrateinduced assembly of fibronectin into networks: influence of surface chemistry and effect on osteoblast adhesion," Tissue Engineering Part A, vol. 15, no. 11, pp. 3271-3281, 2009.

[3] N. Miranda Coelho, C. González-García, J. A. Planell, M. Salmerón-Sánchez, and G. Altankov, "Different assembly of type iv collagen on hydrophilic and hydrophobic substrata alters endothelial cells interaction," European Cells and Materials, vol. 19, pp. 262-272, 2010.

[4] R. O. Hynes, "Integrins: bidirectional, allosteric signaling machines," Cell, vol. 110, no. 6, pp. 673-687, 2002.

[5] C. Chung and J. A. Burdick, "Influence of three-dimensional hyaluronic acid microenvironments on mesenchymal stem cell chondrogenesis," Tissue Engineering A, vol. 15, no. 2, pp. 243254, 2009.

[6] G. C. Wood, "The formation of fibrils from collagen solutions. 3. Effect of chondroitin sulphate and some other naturally occurring polyanions on the rate of formation," Biochemical Journal, vol. 75, pp. 605-612, 1960.

[7] R. Pankov and K. M. Yamada, "Fibronectin at a glance," Journal of Cell Science, vol. 115, no. 20, pp. 3861-3863, 2002.

[8] R. F. Mhanna, J. Voros, and M. Zenobi-Wong, "Layer-bylayer films made from extracellular matrix macromolecules on silicone substrates," Biomacromolecules, vol. 12, no. 3, pp. 609616, 2011.

[9] J. Å. Johansson, T. Halthur, M. Herranen, L. Söderberg, U. Elofsson, and J. Hilborn, "Build-up of collagen and hyaluronic acid polyelectrolyte multilayers," Biomacromolecules, vol. 6, no. 3, pp. 1353-1359, 2005.

[10] N. Aggarwal, N. Altgärde, S. Svedhem, G. Michanetzis, Y. Missirlis, and T. Groth, "Tuning cell adhesion and growth on biomimetic polyelectrolyte multilayers by variation of $\mathrm{pH}$ during layer-by-layer assembly," Macromolecular Bioscience, vol. 13, no. 10, pp. 1327-1338, 2013. 
[11] R. P. W. Rouhl, R. J. van Oostenbrugge, J. Damoiseaux, J.-W. Cohen Tervaert, and J. Lodder, "Endothelial progenitor cell research in stroke: a potential shift in pathophysiological and therapeutical concepts," Stroke, vol. 39, no. 7, pp. 2158-2165, 2008.

[12] C. Kalka, H. Masuda, T. Takahashi et al., "Transplantation of ex vivo expanded endothelial progenitor cells for therapeutic neovascularization," Proceedings of the National Academy of Sciences of the United States of America, vol. 97, no. 7, pp. 3422$3427,2000$.

[13] R. P. W. Rouhl, R. J. Van Oostenbrugge, J. Damoiseaux, J.-W. Cohen Tervaert, and J. Lodder, "Endothelial progenitor cell research in stroke: a potential shift in pathophysiological and therapeutical concepts," Stroke, vol. 39, no. 7, pp. 2158-2165, 2008.

[14] N. Rozen, T. Bick, A. Bajayo et al., "Transplanted blood-derived endothelial progenitor cells (EPC) enhance bridging of sheep tibia critical size defects," Bone, vol. 45, no. 5, pp. 918-924, 2009.

[15] K. Atesok, R. Li, D. J. Stewart, and E. H. Schemitsch, "Endothelial progenitor cells promote fracture healing in a segmental bone defect model," Journal of Orthopaedic Research, vol. 28, no. 8, pp. 1007-1014, 2010.

[16] J. G. Roncalli, J. Tongers, M.-A. Renault, and D. W. Losordo, "Endothelial progenitor cells in regenerative medicine and cancer: a decade of research," Trends in Biotechnology, vol. 26, no. 5, pp. 276-283, 2008.

[17] P. Jiménez-Quevedo, G. V. Silva, R. Sanz-Ruiz et al., "Diabetic and nondiabetic patients respond differently to transendocardial injection of bone marrow mononuclear cells: findings from prospective clinical trials in "no-option" patients," Revista Espanola de Cardiologia, vol. 61, no. 6, pp. 635-639, 2008.

[18] S. F. Mause and C. Weber, "Microparticles: protagonists of a novel communication network for intercellular information exchange," Circulation Research, vol. 107, no. 9, pp. 1047-1057, 2010.

[19] M. C. Deregibus, V. Cantaluppi, R. Calogero et al., "Endothelial progenitor cell derived microvesicles activate an angiogenic program in endothelial cells by a horizontal transfer of mRNA," Blood, vol. 110, no. 7, pp. 2440-2448, 2007.

[20] J. Wang, S. Chen, X. Ma et al., "Effects of endothelial progenitor cell-derived microvesicles on hypoxia/reoxygenation-induced endothelial dysfunction and apoptosis," Oxidative Medicine and Cellular Longevity, vol. 2013, Article ID 572729, 9 pages, 2013.

[21] J. Chen, S. Chen, Y. Chen et al., "Circulating endothelial progenitor cells and cellular membrane microparticles in $\mathrm{db} / \mathrm{db}$ diabetic mouse: possible implications in cerebral ischemic damage," American Journal of Physiology-Endocrinology and Metabolism, vol. 301, no. 1, pp. E62-E71, 2011.

[22] E. J. Marrotte, D.-D. Chen, J. S. Hakim, and A. F. Chen, "Manganese superoxide dismutase expression in endothelial progenitor cells accelerates wound healing in diabetic mice," The Journal of Clinical Investigation, vol. 120, no. 12, pp. 4207-4219, 2010.

[23] Y. Liu, Q. Pan, Y. Zhao et al., "MicroRNA-155 regulates ROS production, $\mathrm{NO}$ generation, apoptosis and multiple functions of human brain microvessel endothelial cells under physiological and pathological conditions," Journal of Cellular Biochemistry, vol. 116, no. 12, pp. 2870-2881, 2015.

[24] H. S. Cheng, N. Sivachandran, A. Lau et al., "MicroRNA-146 represses endothelial activation by inhibiting pro-inflammatory pathways," EMBO Molecular Medicine, vol. 5, no. 7, pp. 949-966, 2013.

[25] H. Ai, S. A. Jones, and Y. M. Lvov, "Biomedical applications of electrostatic layer-by-layer nano-assembly of polymers, enzymes, and nanoparticles," Cell Biochemistry and Biophysics, vol. 39, no. 1, pp. 23-43, 2003.

[26] B. Lapergue, A. Mohammad, and A. Shuaib, "Endothelial progenitor cells and cerebrovascular diseases," Progress in Neurobiology, vol. 83, no. 6, pp. 349-362, 2007.

[27] S. Meng, J.-T. Cao, B. Zhang, Q. Zhou, C.-X. Shen, and C.Q. Wang, "Downregulation of microRNA-126 in endothelial progenitor cells from diabetes patients, impairs their functional properties, via target gene Spred-1," Journal of Molecular and Cellular Cardiology, vol. 53, no. 1, pp. 64-72, 2012.

[28] Y.-H. Chen, S.-J. Lin, F.-Y. Lin et al., "High glucose impairs early and late endothelial progenitor cells by modifying nitric oxide-related but not oxidative stress-mediated mechanisms," Diabetes, vol. 56, no. 6, pp. 1559-1568, 2007.

[29] J. Chen, J. Chen, S. Chen et al., "Transfusion of CXCR4-primed endothelial progenitor cells reduces cerebral ischemic damage and promotes repair in $\mathrm{db} / \mathrm{db}$ diabetic mice," PLoS ONE, vol. 7, no. 11, Article ID e50105, 2012.

[30] J. Chen, X. Xiao, S. Chen et al., "Angiotensin-converting enzyme 2 priming enhances the function of endothelial progenitor cells and their therapeutic efficacy," Hypertension, vol. 61, no. 3, pp. 681-689, 2013.

[31] G. N. Chironi, C. M. Boulanger, A. Simon, F. Dignat-George, J.-M. Freyssinet, and A. Tedgui, "Endothelial microparticles in diseases," Cell and Tissue Research, vol. 335, no. 1, pp. 143-151, 2009.

[32] C. M. Boulanger, "Microparticles, vascular function and hypertension," Current Opinion in Nephrology and Hypertension, vol. 19, no. 2, pp. 177-180, 2010.

[33] P. A. Brogan, V. Shah, C. Brachet et al., "Endothelial and platelet microparticles in vasculitis of the young," Arthritis and Rheumatism, vol. 50, no. 3, pp. 927-936, 2004.

[34] A. Mezentsev, R. M. H. Merks, E. O’Riordan et al., "Endothelial microparticles affect angiogenesis in vitro: role of oxidative stress," American Journal of Physiology-Heart and Circulatory Physiology, vol. 289, no. 3, pp. H1106-H1114, 2005.

[35] V. Cantaluppi, S. Gatti, D. Medica et al., "Microvesicles derived from endothelial progenitor cells protect the kidney from ischemia-reperfusion injury by microRNA-dependent reprogramming of resident renal cells," Kidney International, vol. 82, no. 4, pp. 412-427, 2012.

[36] V. Cantaluppi, L. Biancone, F. Figliolini et al., "Microvesicles derived from endothelial progenitor cells enhance neoangiogenesis of human pancreatic islets," Cell Transplantation, vol. 21, no. 6, pp. 1305-1320, 2012.

[37] B. Qin, H. Yang, and B. Xiao, "Role of microRNAs in endothelial inflammation and senescence," Molecular Biology Reports, vol. 39, no. 4, pp. 4509-4518, 2012.

[38] A. Zernecke, K. Bidzhekov, H. Noels et al., "Delivery of microRNA-126 by apoptotic bodies induces CXCL12dependent vascular protection," Science Signaling, vol. 2, no. 100, article ra81, 2009.

[39] K. S. Tan, A. Armugam, S. Sepramaniam et al., "Expression profile of microRNAs in young stroke patients," PLOS ONE, vol. 4, no. 11, Article ID e7689, 2009. 
[40] S. Fichtlscherer, S. De Rosa, H. Fox et al., "Circulating microRNAs in patients with coronary artery disease," Circulation Research, vol. 107, no. 5, pp. 677-684, 2010.

[41] F. Jansen, X. Yang, M. Hoelscher et al., "Endothelial microparticle-mediated transfer of microRNA-126 promotes vascular endothelial cell repair via spred1 and is abrogated in glucose-damaged endothelial microparticles," Circulation, vol. 128, no. 18, pp. 2026-2038, 2013. 

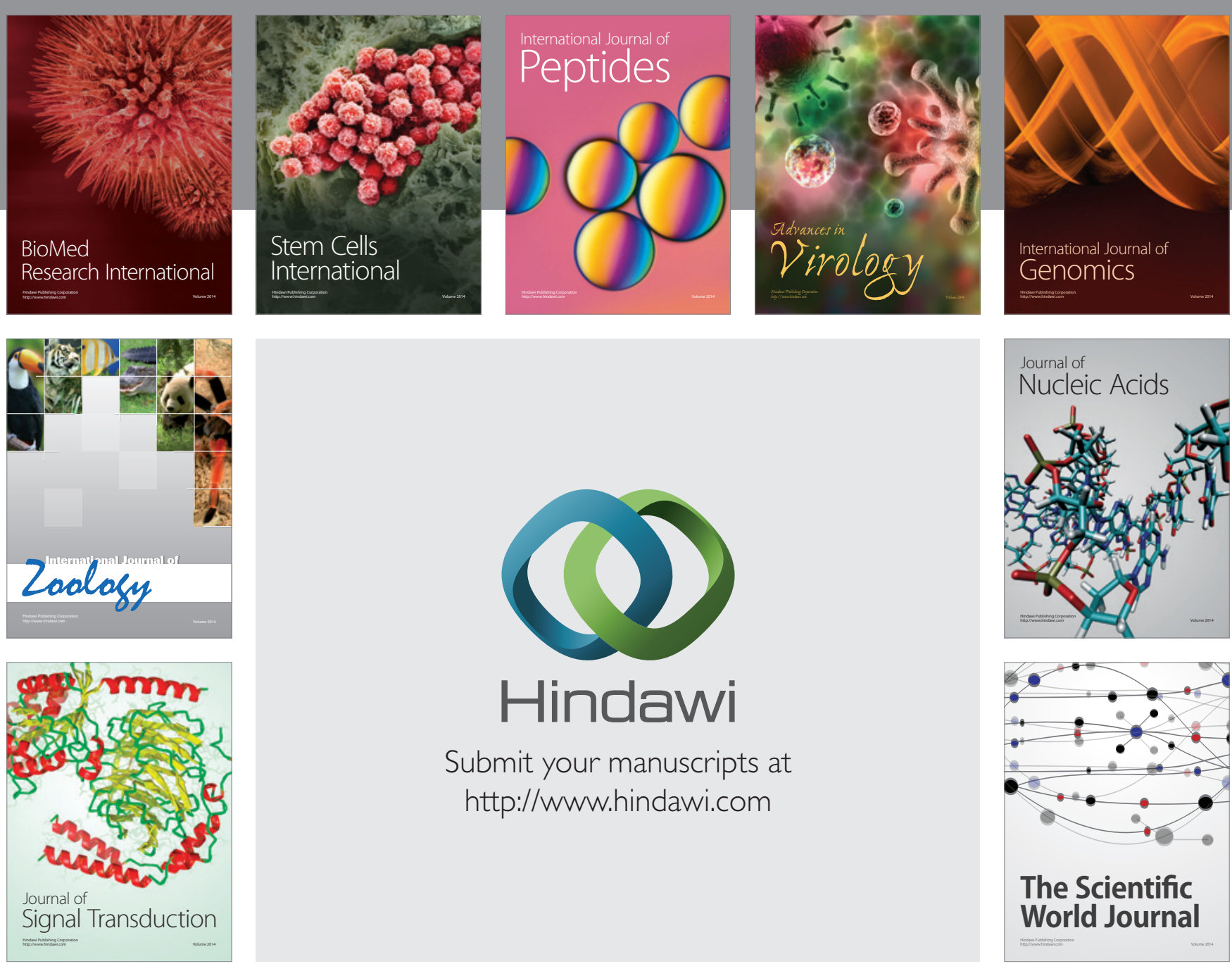

Submit your manuscripts at

http://www.hindawi.com
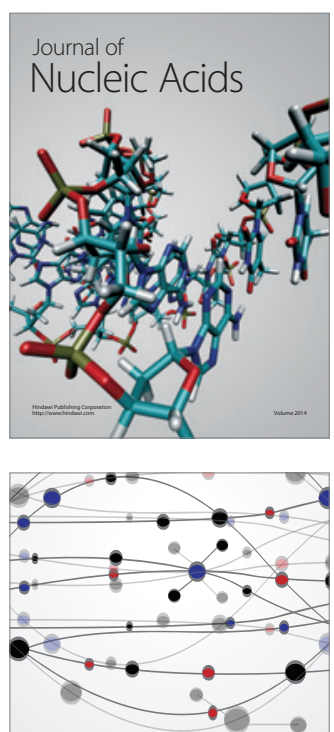

The Scientific World Journal
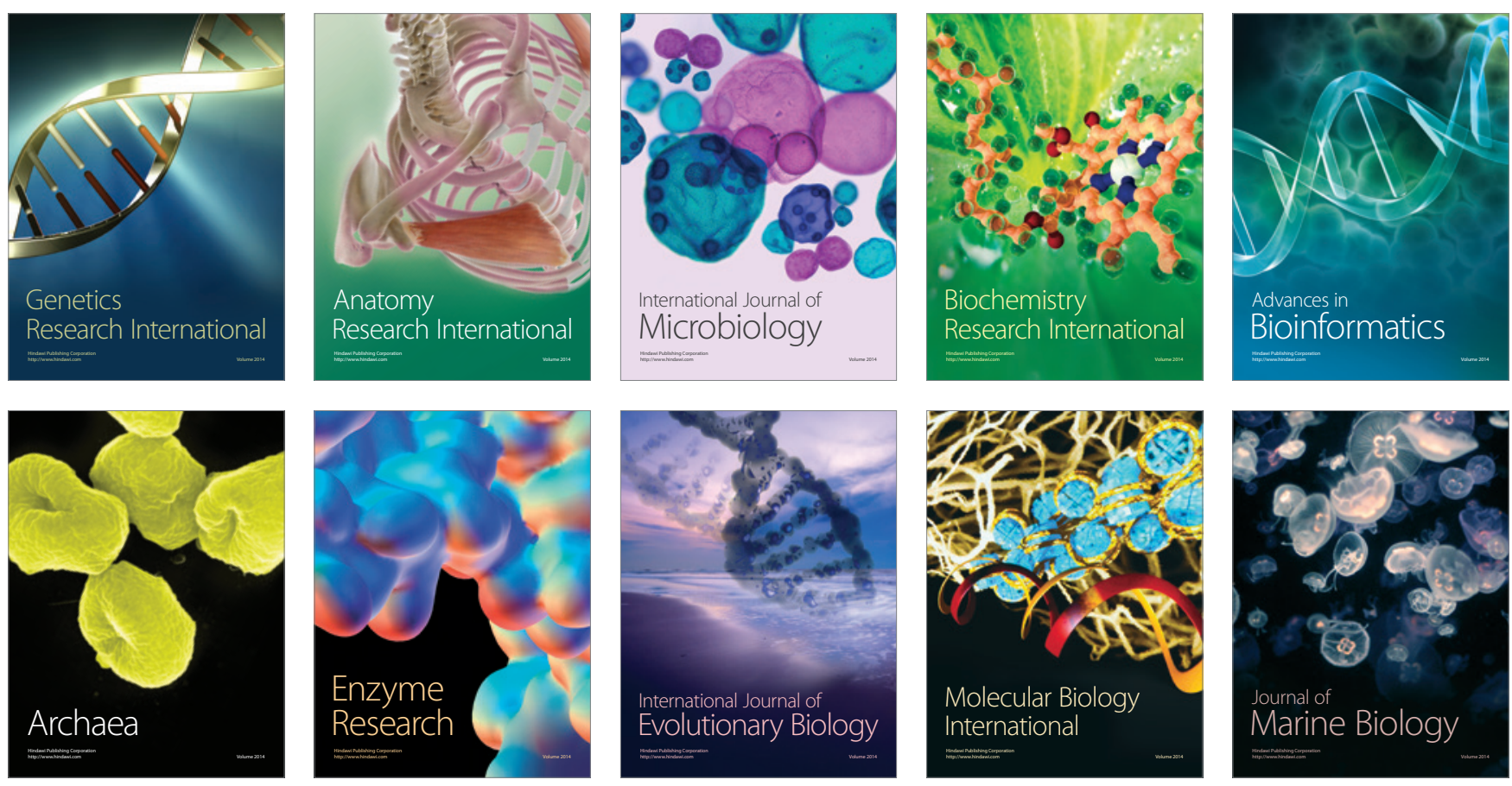